\title{
An EARly Warning System for the Health OF THE OCEANS
}

\author{
By Neil R. Andersen (Editor)
}

\section{$\mathrm{T}$} HE TWO CONVENTIONS signed at the United National Conference on Environment and Development (UNCED, Rio de Janeiro, 1992), The Framework Convention on Climate Change and The Convention on Biological Diversity, and the recommendations of Agenda 21, Chapter 17, require the establishment of an adequate observing system to develop understanding and to monitor change. To do this, continuing systematic, long-term, global observations of marine physical, chemical, and biological conditions, analogous to the World Weather Watch, operating under the auspices of the World Meteorological Organization (WMO), are required. Guided by the Intergovernmental Oceanographic Commission (IOC). WMO, the United National Environmental Program (UNEP) and the International Council for Scientific Unions (ICSU), the planning for the Global Ocean Observing System (GOOS) is being initiated. One aspect of the planning for GOOS is directed at considerations of the health of the ocean. The Health of the Ocean (HOTO) Module of GOOS will provide a basis for the assessment of the state and trends in the marine environment as it is affected by anthropogenic activities, including, inter alia, increased risks to human health, harm to marine resources, alterations of natural change and general ocean health. An international panel was formed under the leadership of the IOC to achieve this goal. The purpose of this document is to outline the strategy and detailed scientific and technical design for the Assessment and Prediction of the Health of the Ocean (HOTO) Module of GOOS. The discussion that follows is an

Neil R. Andersen, Horn Point Environmental Laboratory. Center for Environmental and Estuarine Studies, University of Maryland, P.O. Box 775. Cambridge. MD 21613, USA. overview of deliberations that have taken place over the last three years.

\section{Background}

We face unprecedented pressures on our natural resources. Sustainable development of these resources is hindered by an inability to detect emerging environmental problems at an early stage when remedial measures can still be effective. Nowhere is this inadequacy so pronounced as in the marine environment. Global energy cycles and the biological processes upon which all life depend are critically influenced by the ocean. Knowledge of the ocean and humanity's impact on it is only now beginning to reveal the complexity and interdependence of all aspects of the system. Improved knowledge and predictive capabilities are required for more effective and sustained development of the marine environment to reap associated economic benefits and to preserve marine resources.

The two conventions signed at the United Nations Conference on Environment and Development (UNCED, Rio de Janeiro, 1992), The Framework Convention on Climate Change and The Convention on Biological Diversity, and the recommendations of Agenda 21, Chapter 17. require the establishment of an adequate observing system to develop understanding and to monitor change. Many of the processes that control the variability and change of global climate are themselves controlled by processes in the ocean. Public perceptions of risk are only eased when governments are seen to be keeping the environment, including the ocean. under close observation. If the UNCED goals of global sustainable development and integrated oceans management are to be achieved, a much more integrated data management system and a biological distress signal detection program must be developed. To do this, continuing systematic, long-term, global observations of marine physical, chemical, and biological conditions, analogous to the World Weather Watch, operating under the auspices of the World Meteorological Organization (WMO), are required.

The scope and purpose of such an effort mandate a sound scientifically based strategy and long-term international commitment. Many regular observations of the ocean are done by research programs with limited funding, finite duration, and single objectives, such as the global climate change research programs. Other observations are made by operational agencies for specific purposes but are in limited parts of the world ocean, typically have only sparse coverage, and usually concentrate on the upper ocean and sense a limited number of variables. The requirements of the customers of such observations demand global coverage, including the polar regions, and scientifically designed, robust, cost-affordable, long-term, routine and systematic observations. Most importantly, such an activity must be based on the principle of timely, full and open access to ocean data.

This document outlines the strategy and detailed scientific and technical design for the Assessment and Prediction of the Health of the Ocean (HOTO) Module of the Global Ocean Observing System (GOOS). An international panel was formed under the leadership of the Intergovernmental Oceanographic Commission (IOC) to achieve this goal, drawing heavily on experience gained in implementing the IOC/United Nations Environment Program (UNEP)/International Maritime Organization (IMO) Global Investigation of Pollution in the Marine Environment (GIPME; See IOC 1976 and 1984). This discussion is an overview of 
deliberations that have taken place over the last three years.

\section{The Concept of the Global Ocean Observing System (GOOS)}

The objective of the GOOS is a permanent system of global and systematic observations adequate for forecasting climate variability and change: for assessing the health or state of the marine environment and its resources, including the coastal zone; and for supporting an improved decision-making and management process, which takes into account potential natural and man-made changes in the environment and their effects on human health and resources.

The GOOS is an internationally coordinated system for systematic operational data collection (measurements), data analysis. exchange of data and data products, technology development, and transfer. GOOS will use a globally coordinated. scientifically based strategy to allow for mon- itoring and subsequent prediction of environmental changes globally, regionally. and nationally. Data will be generated by repeat sampling and remote sensing using sea-surface and subsurface instrumentation in the open sea and in coastal regions worldwide. including enclosed and semienclosed areas. Major physical. chemical, and biological variables have been identified by the HOTO Panel that can be used to provide an integrated assessment of the current health of the oceans and early warning of deterioration.

Guided by IOC, WMO, UNEP, and the International Council for Scientific Unions (ICSU), the planning for GOOS is taking place within five specific areas:

- climate monitoring. assessment, and prediction:

- monitoring and assessment of living resources:

- monitoring the coastal environment and its changes:
- assessment and prediction of the health of the ocean; and

- marine meteorological and operational services.

The GOOS is a set of activities that constitutes operational oceanography and its related efforts, including some directed and applied research. GOOS is Oceanography for societal and economic purposes (see Fleming. 1995). coordinating and fostering ocean operations and applications that are long-term, routine, globally relevant, scientifically based, systematic, and affordable.

The GOOS is not a research program but builds on research, it provides data for research, and supporting directed and applied research is a significant contribution to GOOS. Academic institutions have provided the foundation upon which GOOS is built, through research programs. technology development. and training of human resources. A strong

\title{
What DOES GOOS/HOTO MEAN, AND What WiLl IT OFFER, TO THE MARINE SCIENTIST?
}

\author{
By Herbert L. Windom
}

$\mathrm{T}$ HE IMPLEMENTATION of the Health of the Oceans Module (HOTO) of the Global Ocean Observing System (GOOS) is intended to occur. region by region. in concert and ideally amalgamated, with the other GOOS components, particularly the climate, living resources, and coastal zone modules. However, what HOTO potentially offers to the marine scientist is a scheme of continuing measurements accomplished in a more comparable manner. that can be used by individuals for their own applications. The global development of HOTO is through national and regional implementation activities that address identified problems in specific geographic areas and assess their extent in time and space. Individual scientists, therefore, should seize the opportunity of becoming involved in the planning and implementation of such specific monitoring activities. HOTO data will be made available in raw form and in a range of collated and interpreted forms that will be useful for a suite of marine and nonmarine applications. HOTO also will provide a mechanism for obtaining a measurement series on which individual scientists can base, or piggyback, any specific measurement requirements that they have for scientific and/or management applications. This then is the promise of HOTO. It is now up to the community involved in the design of the several GOOS modules to complete their work and to use the international science, environmental management, and policy communities to ensure the implementation of all the modules in a systematic and coordinated manner. The entire sequence of operations within GOOS, if implemented as intended, will provide a vastly improved store of basic marine environmental data with higher spatial and temporal resolution than available ever before. This will mean that individual scientists can reduce the effort they have to devote to routine measurements and concentrate their efforts on the necessary incremental measurements for their own purposes.

Herbert L. Windom, Skidaway Institute of Oceanography. Savannah, Georgia 31411. USA. 
continued partnership between governments and universities is essential. Applications suggest new research areas and new research results suggest new applications.

\section{Present Status of GOOS}

GOOS has been developing over the past 6 years. Efforts have included identification of needs, consultation with user groups, assessment of what is technologically possible, identification of existing scientific knowledge. identification of future scientific objectives, assessment of present routine and/or operational observations, assistance and capacity building for developing countries. and the development of models and requirements for output data products.

The GOOS Strategic Plan (IOC, 1995) agreed to at I-GOOS-II and endorsed by Resolution 11 of the IOC AssemblyXVIII (13-27 June 1995. Paris) will produce a road map for GOOS and will identify the priorities for the essential work needed to develop and implement GOOS. A Priorities Agreement Meeting for GOOS participants is an essential next step in the process and will be convened in early 1997. The basic purpose of this GOOS Priorities Agreement Meeting is for all the participants in GOOS to indicate those activities that they agree are important to maintain now. and to discuss and allocate priorities to future operations.

At the present time only two modules of GOOS have received significant attention; the Climate Module (see OOSDP, 1995) and the Health of the Ocean (HOTO) Module.

\section{Purposes and Benefits of the HOTO Module}

The HOTO Module of GOOS provides a basis for the assessment of the state and trends in the marine environment as it is affected by anthropogenic activities, in cluding. inter alia, increased risks to human health, harm to marine resources, alterations of natural change, and general ocean health.

The term "Health of the Oceans" is operationally defined for the purposes of the HOTO Module of GOOS as a reflection of the condition of the marine environment from the perspective of adverse effects caused by anthropogenic activities. in particular habitat destruction. changed sedimentation rates, and the mobilization of contaminants. Such condi- tion refers to the contemporary status of the ocean. prevailing trends, and the prognosis for improvement or deterioration in its quality.

Although the measurement of contaminant loads can and must be measured. such measurements by themselves cannot provide comprehensive quality criteria. Biological indices will have to be identified at four levels: suborganismal, individuals, populations. and communities. Molecular, cellular, physiological and behavioral disturbances, and pathological manifestations will be needed to reflect responses at the individual organism level. At the population and community levels. effects might manifest themselves in changes in the reproductive success of species, the disruption of the dynamic balance between producers and consumers, or deviations from the natural range of biomass variability leading to abnormal phytoplankton blooms or monospecific swarms. Critical habitats. such as coral reefs, estuaries, temperate and tropical wetlands including mangroves, submerged macrophyte communities, and other spawning and nursery areas will require identification of more specific biological indices. In all cases, biological indices will have to be assessed against the background of natural variability.

\section{Relationships Between HOTO Indicators, Global Ocean Health, and Sustainable Development}

The kind of systematic and global monitoring to be carried out under HOTO will provide a broad range of critical benefits in two broad, but clearly interrelated, categories. Tables 1 and 2 illustrate the ways in which the HOTO Module can contribute directly to (1) our general understanding of the present status and future trends in global ocean health and (2) the ability of State and National governments to maximize socioeconomic benefits derived from sustainable development and the use of ocean/ coastal areas.

A primary contribution of the HOTO Module is to develop a global perspective of the impact of human activities on ocean health. This kind of long-term monitoring strategy will contribute directly to a more basic understanding of marine ecosystem health, human health implications of regional and global deterioration of the oceans, the ability of States to achieve goals in sustainable development, and the influence of the oceans on global climate change. A general characterization of the relationships between the variables to be monitored under the HOTO Plan and measures of global ocean health is presented in Table 1. The respective assignments of the entries " 3, " "2," and " 1 " signify increasing strengths in the relationships among the variables and the issues. Blank cells represent either a weak relationship or one in which current scientific information is too limited to make an assignment.

The increase of radiatively active, or so-called "greenhouse" gases in the atmosphere could lead to a change in climate, although not specifically indicated in Table 1. This topic is within the purview of the Climate Module of GOOS, but some effects of climate change need to be considered in the development of the HOTO Module. These effects could include changes in the frequency and diversity of toxic algal blooms, changes in nutrient influxes and oxygen conditions in coastal areas. altered influxes of suspended matter. and changes in atmospheric precipitation patterns. The role of carbon dioxide is being addressed by major research programs addressing climate change such as the Global Climate Research Program (GCRP) and the International GeosphereBiosphere Program (IGBP).

\section{Biological Health}

Key objectives of the HOTO Plan are to provide the information necessary to ensure the maintenance of biodiversity and the integrity of marine communities, minimize the loss of species. limit human influences on living marine resources (including genetic richness). protect critical habitats, and safeguard human health.

\section{Relationships between HOTO Indicators, Human Uses, and Socioeconomic Factors}

In addition to making a fundamental contribution to our global, long-term understanding of trends in global ocean health, the HOTO Module can provide critical insights into the development of management strategies designed to maximize the benefits of sustainable marine resource use. Table 2 provides a general assessment of the nature and scope of the relationship between marine resource/use benefits and certain contaminant/analyte 
Table 1

Relationship between global ocean health. contaminants/analytes. and sustainable development

VARIABLES

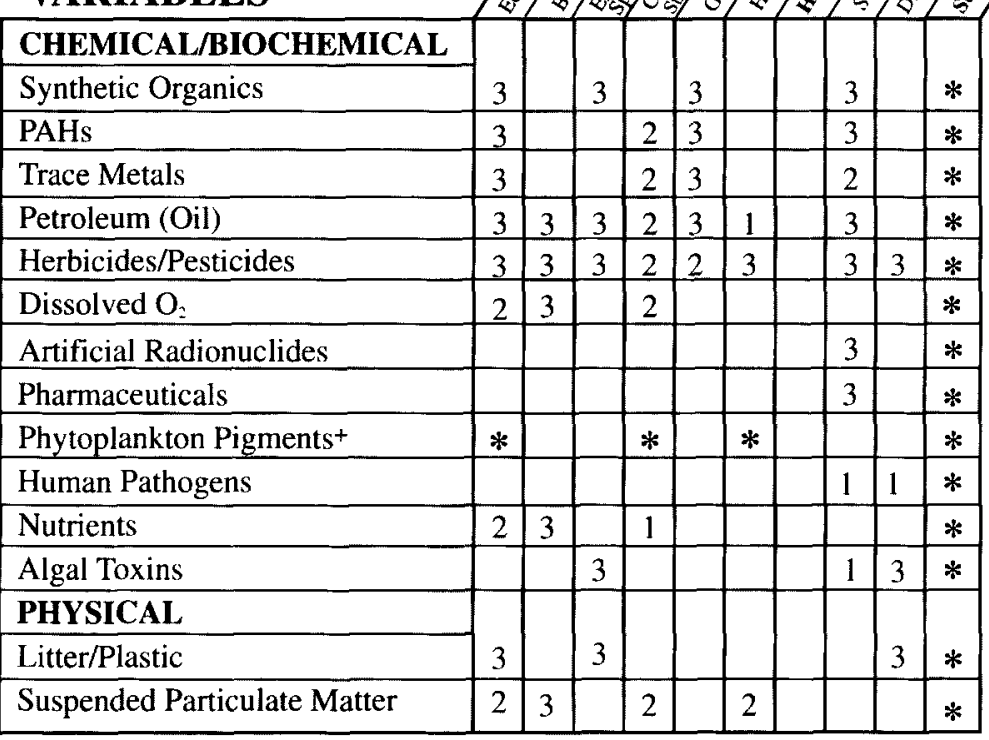

1 - Strong 2-Moderate 3-Low *Directly Related

+ Phytoplankton prgments may serve as indicators of global ocean health. classes. As in Table 1. the numbers 3, 2. and 1 denote increasing importance, and the arrows signify the direction of impact. Blank cells represent either a weak relationship or one in which current scientific information is inadequate to make an assignment.

\section{Specific HOTO Measurements Required}

The problem areas identified in the preceding section can be best addressed through the use of appropriate suites of physical, chemical, and biological measurements. Here, the measurements se-
Table 2

Impact on socioeconomic benefits in the coastal zone

\begin{tabular}{|c|c|c|c|c|c|c|c|c|c|c|c|c|c|c|c|c|c|c|c|}
\hline VARIABLES & 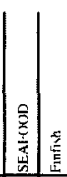 & $\frac{\bar{n}}{\overline{\underline{\underline{n}}}}$ & $\mid \begin{array}{l}\frac{y}{2} \\
\frac{3}{3} \\
\frac{5}{3} \\
\end{array}$ & 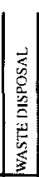 & & & 离 & & 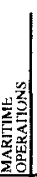 & 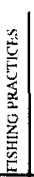 & & & 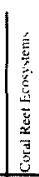 & 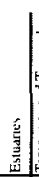 & 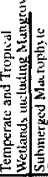 & & & & 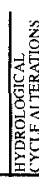 \\
\hline $\begin{array}{l}\text { CHEMICAL/BIOCHEMICAL } \\
\text { Synthetic Organics }\end{array}$ & $\cdot 3$ & 312 & $\cdot 3$ & & 3 & i & $\frac{1}{2}$ & & 3 & & & & & 2 & +3 & & 3 & & \\
\hline PAHs & 13 & $3+2$ & 13 & & $\dot{2}$ & 2 & $\overline{3}$ & & 3 & & i & & & +2 & & & 3 & 3 & \\
\hline Trace Metals & +1 & \begin{tabular}{l|l}
12 \\
\end{tabular} & 43 & & $\overline{2}$ & i & 2 & & 3 & & [ & & & $i^{\circ}$ & & & 2 & $\dot{2}$ & \\
\hline Petroleum (Oil) & $\cdot 3$ & $3: 1$ & $i 3$ & & $i$ & 2 & 3 & 11 & $i^{2}$ & & $i$ & & 43 & +2 & 114 & $3+$ & & & \\
\hline Herbicides/Pesticides & +2 & \begin{tabular}{l|l}
2 & 12 \\
2 & -1
\end{tabular} & $2 \cdot 3^{2}$ & & 2 & 3 & i & 2 & 3 & & & & 12 & $3^{2}$ & $12+$ & 2. & & 1 & \\
\hline Dissolved $\mathrm{O}_{2}$ & 11 & $1 \cdot 1$ & .6 & & $i$ & $\overline{1}$ & $\frac{1}{2}$ & +3 & 3 & & 3 & & & +1 & $13:$ & $3:$ & 2 & 3 & 3 \\
\hline Artificial Radionuclides & & & & & & $i$ & & & 3 & & & & & & & & . & & \\
\hline Pharmaceuticals & & & 2 & & 3 & 2 & 3 & & & & & & & & & & & & \\
\hline Phytoplankton Pigments & & & & & 2 & 3 & 1 & $2^{3}$ & & & & & 2 & & $i$ & 3 & 3 & $\div 1$ & 11 \\
\hline Human Pathogens & 11 & 111 & .1 & & i & & & 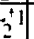 & 3 & & & & & & & & & & \\
\hline Nutrients & & & $2^{3}$ & & it & 3 & i & :3 & 3 & & & & 12 & & & & $\cdot 3$ & it & 1.1 \\
\hline Algal Toxins & +1 & $1+1$ & $1 \%$ & & & & & 1 & & & & & 3 & & & & & & \\
\hline $\begin{array}{l}\text { PHYSICAL } \\
\text { Litter/Plastic }\end{array}$ & +3 & 3 & -3 & & $i$ & $i$ & ${ }_{3}$ & 少 & $i^{3}$ & 3 & & & & & & & & 3 & \\
\hline Suspended Particulate Matter & & +3 & $\sqrt{3} 3$ & & i & $\ddot{2}$ & $i$ & $3^{12}$ & 1 & 3 & 3 & 2 & $\$ 1$ & 52 & $i$ & $3 t$ & 3 & $i$ & $i$ \\
\hline
\end{tabular}

lected for inclusion in the HOTO Module are specified in two categories: 1) chemical, biochemical, and physical analytes; and 2) biological effects measurements and indicators of ecosystem health.

\section{Chemical, Biochemical, and Physical Analytes}

The following set of basic variables, which have been selected for inclusion in HOTO, correspond both to the variables identified in the analyses depicted in $\mathrm{Ta}^{-}$ bles 1 and 2 above and those identified as priority contaminants within the Global Investigation of Pollution in the Marine Environment (GIPME) Program (IOC, 1976 and 1984) and in the UNEP Regional Seas Action Plans (UNEP, 1991).

\section{Biological Effects Measurements}

The HOTO Module will employ simultaneous monitoring at different levels of biological organization to provide a better understanding of the mechanistic links among changes at each level (see Fig. 1 and Table 3). This will also aid in the assessment of the effectiveness of management and mitigation measures for dealing with contaminant disposal and degradation. A further advantage of this strategy will be the potential to assess the effectiveness of management and mitigation measures dealing with contaminant disposal.

Changes at the population/community/ecosystem levels of biological organization are of ultimate concern. However, such high-level responses are generally complex and far removed from causative events and are manifestations of damage rather than predictive indices. Detection of lower level changes (e.g., molecular, cellular, physiological, and behavioral responses) that underlie higher level effects and for which causality can be established, may provide early warning of impending environmental damage. Individual and subindividual responses may also be more amenable to detection by automated monitoring systems.

To ensure global applicability of methodology in different regions of the world and to ensure uniform interpretation of results, the use of biological techniques at the organismic and suborganismic levels (e.g., biomarkers, physiological techniques, etc.) requires the prior identification of related/ analogous members of a given biological group (not necessarily the same species) considering climatic/geographical variations and differences, but corresponding in genera, 
family, or biological class. Such use of analogous members would provide a uniform and reliable global picture of particular analytes/contaminants/situations under consideration. Furthermore, this will also enhance the global relevance of such techniques.

\section{Monitoring Considerations}

The HOTO Panel stressed that it is essential that the goals and objectives of any monitoring program be clearly stated from the outset. The entire design of a program should flow from these goals; no monitoring program can measure every variable or address every management issue. Once the goals and objectives are clear, the sampling design must be tailored to meet them. In particular, it is imperative to specify the level of change anticipated or directed by policy objectives, in quantitative terms, the minimum magnitude of change or effect that the program must be able to detect. and to ensure that the design has adequate statistical power to accomplish this.

The statistical design must allow assessment of both the spatial and temporal scales of environmental effects. In relation to the monitoring of chemicals: 1) the relationship between measurements and matrices, 2) the relationships between difficulty of measurement and the importance of the measurement, and 3) temporal and spatial aspects of contaminant monitoring have to be considered. In addition, this approach must be tested by an evaluation of the variables in the context of their relative regional importance.

In making decisions regarding sampling in new localities, ordination techniques can be used to rank the status of study sites relative to one another and with respect to conditions at sites considered to represent background conditions. However, for successful managerial action, temporal (i.e., longitudinal) sampling series are advisable so that patterns and trends in anthropogenic stressors, contaminant levels, and associated biological effects can be monitored. Such sampling permits the consequences of managerial actions adopted to ameliorate adverse environmental consequences of anthropogenic activities to be evaluated. Usually, a significant proportion of study sites should be located in the vicinity of expected contaminant sources (e.g.. in the vicinity of river discharges and urban harbors) to maximize the signal-to-noise ratio of measurements. In cases where at- mospheric transport is the primary agent of contaminant delivery to the area concerned, measurements should be made of this input. Recognizing relationships between anthropogenic stressors, contaminant levels, and associated biological responses is a prerequisite to the prediction of future environmental changes.

\section{Statistical Design Criteria}

Once the objectives of the implementation plan in a given region have been formulated, attention must be given to sampling design. If no data are available. pilot studies will be needed to assess the scales of spatial and temporal variability in the analytes measured.

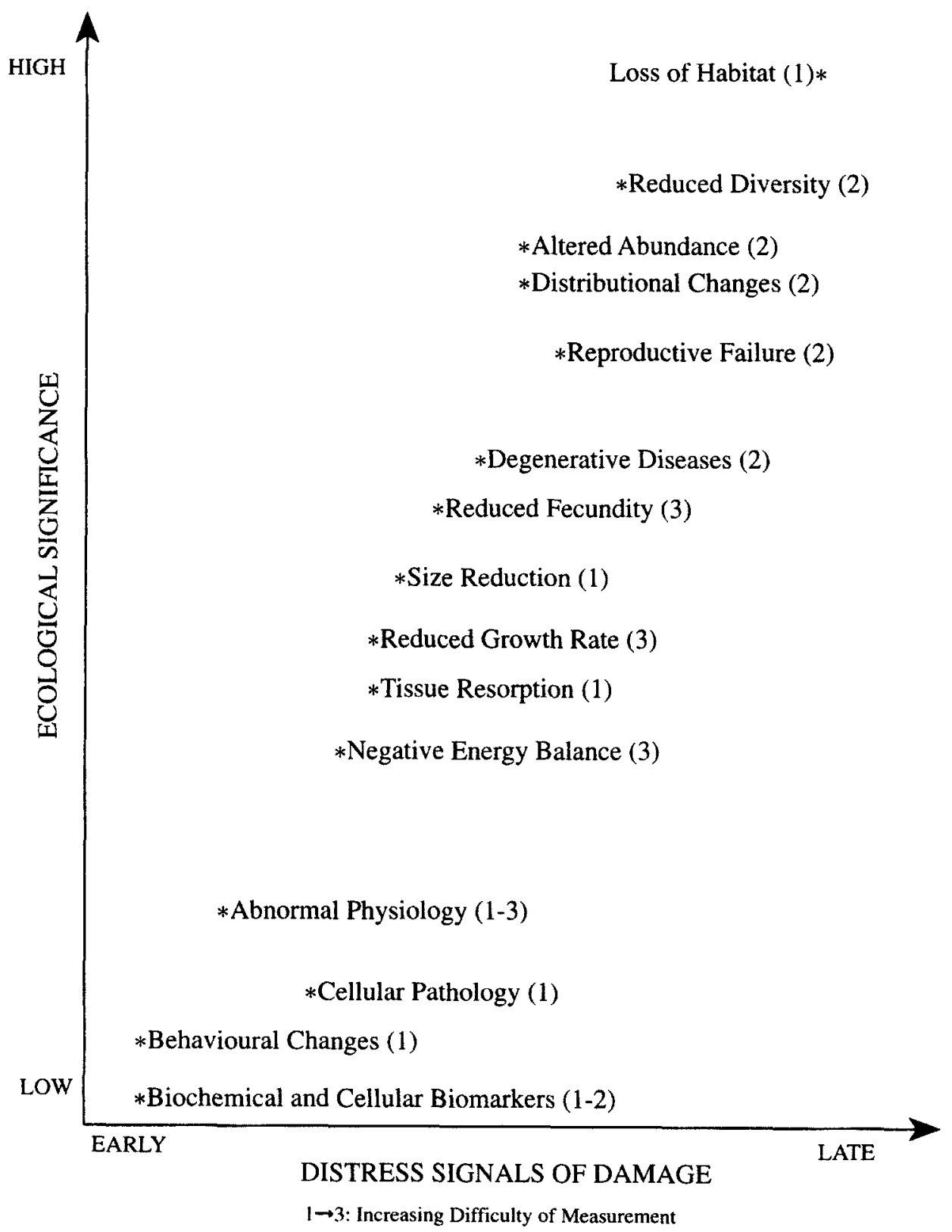

Fig. 1: Biological distress signals of damage to ocean health.
In the past, sampling designs have concentrated on reducing statistical type-I errors (i.e., the risk of rejecting a null hypothesis when it is true). This is done by setting the probability of rejecting the null hypothesis at a value of $p=$ 0.05 . Yet, if one reduces a type-I error, one increases the risk of committing a type-II error (i.e., the risk of accepting a null hypothesis when it is false). This is particularly relevant for HOTO considerations. For example, if one does a statistical test, such as a " $t$-test," on mean concentrations of two samples of heavy metals in fish consumed by man and finds no significant difference, this may simply be due to the sampling design
*Altered Abundance (2)

*Reproductive Failure (2)

*Degenerative Diseases (2)

$*$ Reduced Fecundity (3)

Size Reduction (1)

Reduced Growth Rate (3)

*Tissue Resorption (1)

Negative Energy Balance (3)

$1 \rightarrow 3$ : Increasing Difficulty of Measurement 
Table 3

Contaminant and stress-related signals of harmful biological effects in individuals

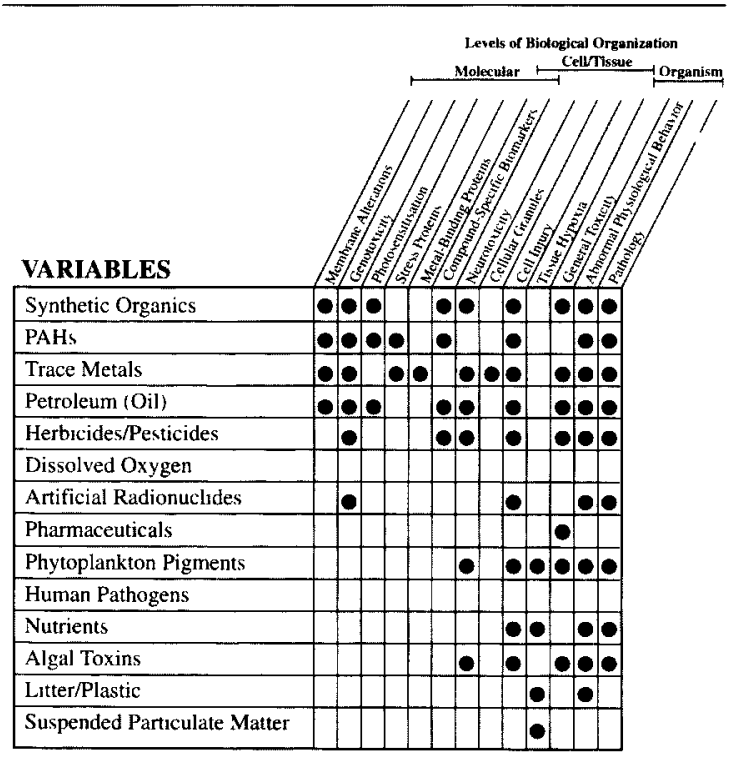

being too low power ever to detect such a difference. In such cases, one must quote the power of a particular test to detect a change (Peterman, 1990; Fairweather, 1991). Power analyses are highly relevant to management issues where managers can decide a priori what magnitude of change they are concerned with. Then it is possible. using power analyses, to design a sampling program to detect such changes.
In addition to the incorporation of power analyses in the design of sampling programs, there is a need to consider other new approaches such as Beyond Before-After Control-Impact (Underwood, 1991, 1992). Here, emphasis is placed on multiple controls and random sampling over time in order to distinguish effects of impacts over natural variability.

Feedback monitoring (Gray and Jensen, 1993), which tests predictions

Table 4

Measurement phases for contaminants/analytes

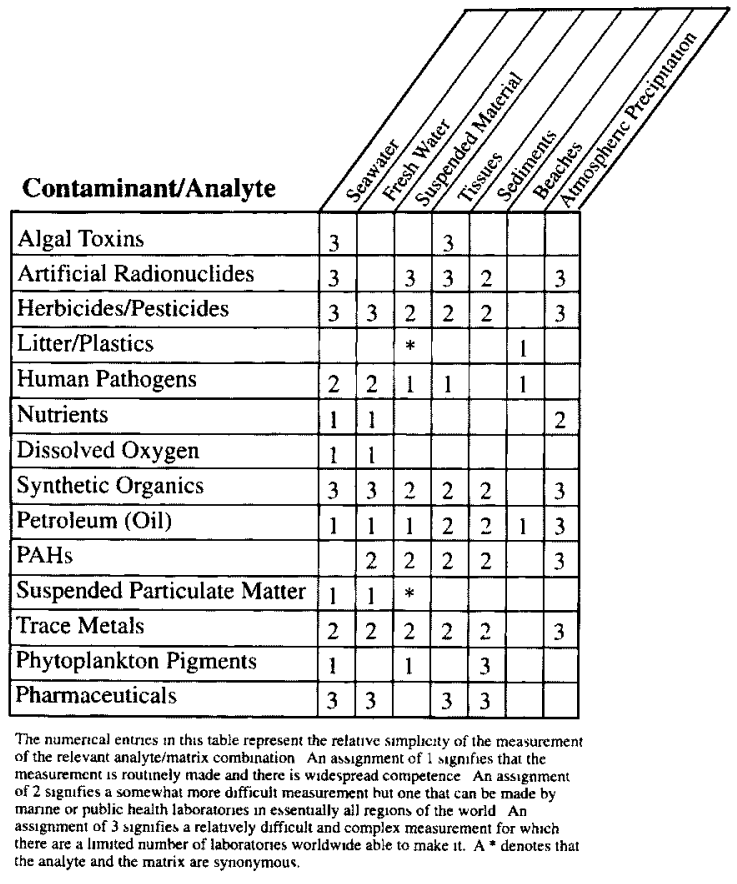

made in monitoring programs, can play an important role in the management of the marine environment.

\section{Measurements Versus Matrices}

The Panel identified the matrices where many of the analytes/contaminants are measured and presented an evaluation of the present state of measurement in these matrices (Table 4). Three categories of difficulty were determined. The first are relatively easy measurements that can be carried out with routine technology. These have been assigned a "1." The second group were defined by the Panel as those requiring more specialized equipment or training but could be analyzed by monitoring laboratories within most nations. These have been assigned a "2." The third group require more sophisticated measurements that would most probably be made by experienced research laboratories and do not exist in all countries. These have been assigned a "3." The matrices chosen were seawater, groundwater and freshwater, suspended particulate matter, biological tissues (i.e., fish/seafood), and sediments. The Panel also added beaches - to represent the fact that litter and oil spills (i.e.. petroleum) impact these areas. Precipitation was also added because this is a mode of entry to the marine environment of some of the analytes discussed above.

\section{Difficulty of Measurement Versus Impact}

The Panel evaluated the analytes/contaminants for degree of impact versus difficulty of measurement. This evaluation drew on an analysis of regional concerns conducted during the Seventh Session of GIPME in 1991 (see IOC, 1991), which took advantage of the experience gained by the GIPME Expert Groups in relation to both measurement capabilities and issues of concern within regional marine areas. As discussed in the previous section, different matrices present different challenges. However, the analysis is represented in Figure 2. The analytes fall into three distinct categories.

Category 1 analytes are those judged both to have high impact and be relatively easy to measure, such as nutrients. pathogens, suspended particulate matter, and plant pigments. Also included in this category are ancillary properties for the characterization of the sampling regime such as salinity, temperature, and dissolved oxygen. Litter and petroleum also have a high impact near sources (spills and dump 


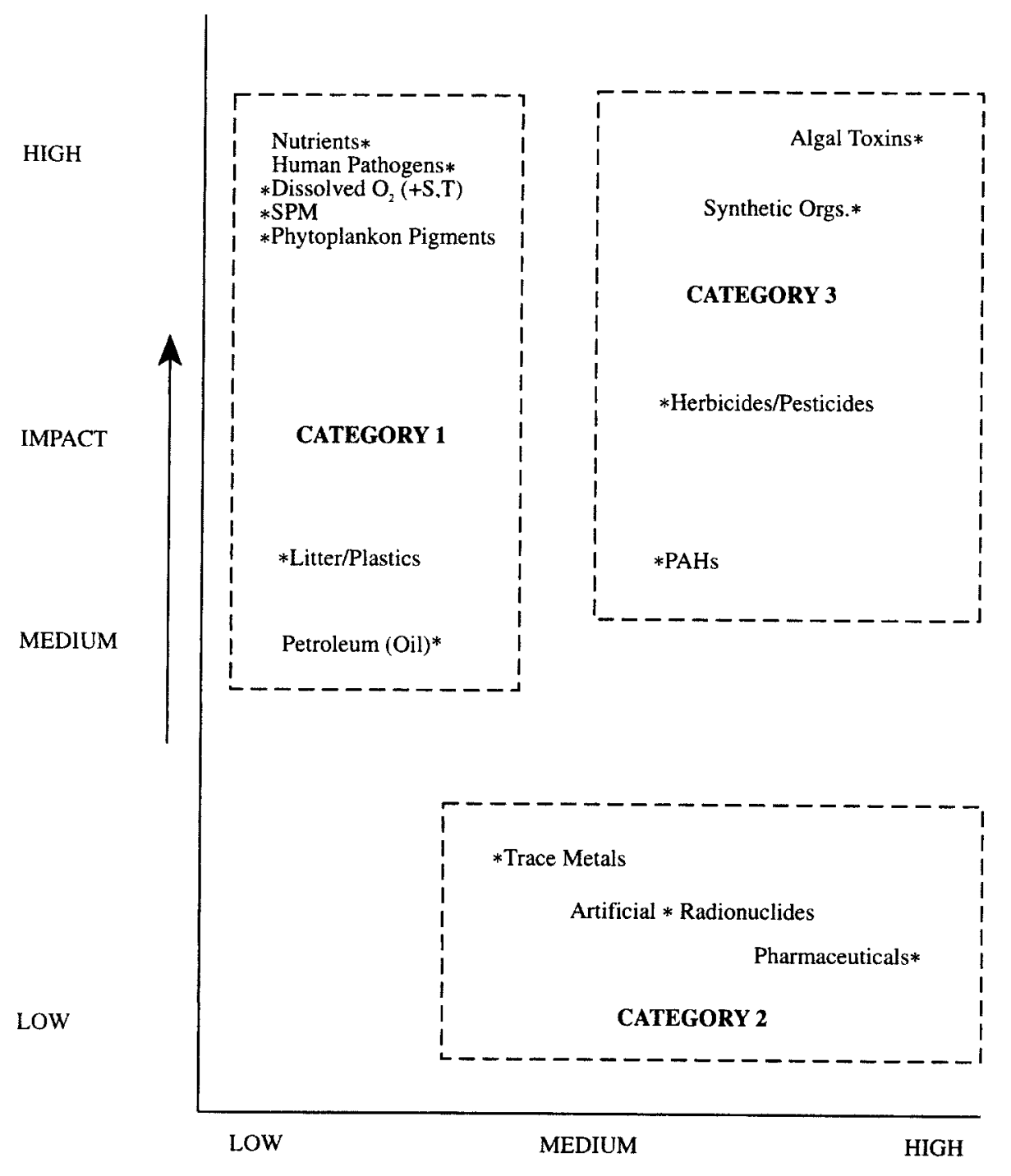

DIFFICULTY OF MEASUREMENT

Fig. 2: Impact versus difficulty of measurement for contaminants/analytes.

sites) as well as at some distance from the sources. These are relatively easy to measure and also belong to Category 1 . These analytes are also judged to be useful for international training programs under, for example, IOC's Training, Education and Mutual Assistance (TEMA) Program.

Category 2 analytes were judged to be difficult to measure and have a lesser impact (i.e., trace metals, artificial radionuclides, and pharmaceuticals). These analytes require sophisticated instrumentation. considerable training, and specialized standards and reference materials. It is worth noting here that the Panel's conclusion is contrary to public perceptions of the impact of these compounds. However, many nonspecialists who consider this issue have a different perception because they often mental conditions are required for local, regional, and global purposes. For local conditions, the highest spatial and temporal resolution will be required. Such resolution is reduced for regional requirements and reduced further for global purposes.

2. Scientific Considerations: The scales of change resulting from anthropogenic introductions of contaminants are an important consideration in selecting spatial and temporal frequencies of measurement. The spatial and temporal resolution required to detect and understand conditions and change will be greatest near to sources and lowest furthest from the sources. This aspect of measurement frequency is further discussed below. For contaminants having natural components, it will be necessary to define the envelope of variability against which anthropogenically induced change can be detected. This will be contaminant specific but likely to impose higher spatial and temporal measurement frequencies in coastal areas than in regional and deep ocean areas.

3. Scales of Natural Variability: Ease of measurement of biological variables is compared with their ecological significance and rate of response in Figure 1. Easy to measure indices at lower levels of the functional and organizational hierarchy offer the best means of obtaining an early warning of deterioration in ocean health. However, with regard to assessing the current extent of habitat loss, measures of community/ecosystem status offer greater potential. A further consideration is that the signal-to-noise ratio will tend to be generally higher at the lower organizational levels (i.e., molecular, cellular, and physiological) and will decrease as the ecological significance of the measurements increases. However, biological communities are naturally variable in both time and space; hence any measurement strategy must take into account, for example, natural, seasonal cycles and spatial patchiness.

Figure 3 attempts to define measurement intervals (i.e., the inverse of measurement frequencies) appropriate for monitoring different contaminants in the context of distance from their sources. It has been formulated on the basis of scientific considerations relating to the likelihood and value of making measurements of change for local human health protection purposes, for example, and for detecting change at locations more remote from sources. 


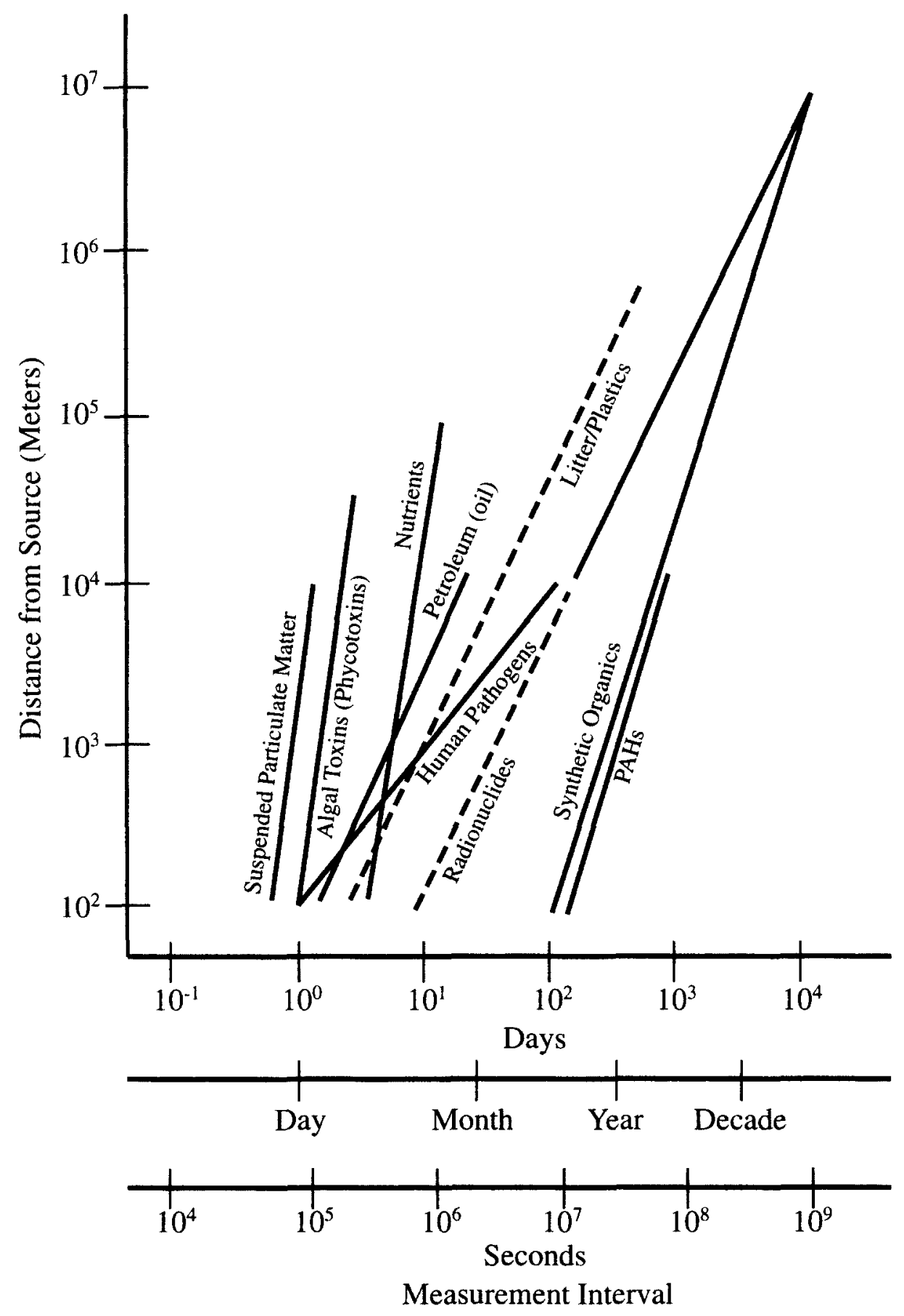

Fig. 3: Spatiotemporal aspects of monitoring of contaminants for health of the oceans assessments.

All Monitoring Networks Should Use the Simplest Methods Available

If capability exists in a region to employ more sophisticated techniques and instrumentation. these should also be used. However, under no circumstances should the simpler techniques be forsaken because of the presence of the capability to employ more sophisticated alternatives.
Harris, P. Haydock. J. Howard and G. Cangelosi. unpublished data.). Continued development of these techniques, together with research into how best they can be applied. is a major aim of the HOTO Module. Increasing the cost-effectiveness of marine observations will be fostered and the production and quality of data will also be enhanced. This will, in turn. necessitate the development of rapid data handling and interpretation systems so that tangible benefits can be derived rapidly from the data generated.

There needs to be continuous evaluation of technical developments and, where appropriate, integration of new techniques for assessing biological effects into the HOTO Module. As temporal changes in the nature and quality of physical, chemical, and biological measurements occur, research will be needed to provide insight into the relationships between the different types of measurements, if the continuity of monitoring is to be maintained.

For biological response measurements, the identification of sensitive indices that can be mechanistically linked to specific contaminant exposures is essential. This would then permit evaluations of the potential of new technologies for making more frequent, more ubiquitous. and more synoptic measurements. Technologies include development of sensors, remote sensing, unattended moorings with or without real-time data telemetering, and autonomous underwater vehicles. Technologies showing most potential for biological response measurement include remote methods of assessing community structure, density, and diversity, on-line. computer-aided physiological and behavioral monitoring systems, molecular and cellular biomarkers of exposure and damage. as well as molecular diagnostic tests such as immunofluorescence detection of proteins and polymerase chain reaction (PCR) for DNA amplification, for example, to detect viable but nonculturable pathogens. Currently, limitations in the measurement of biological responses in the marine environment is the most severe impediments to the implementation of the HOTO Module.

Systems that exist could be improved such as networks of marine laboratories. existing time-series programs in the coastal ocean, and mussel watch activities. New camera systems for benthic surveys. remote submarines, and networks of online biological sensors near major point 


\section{GOOS}

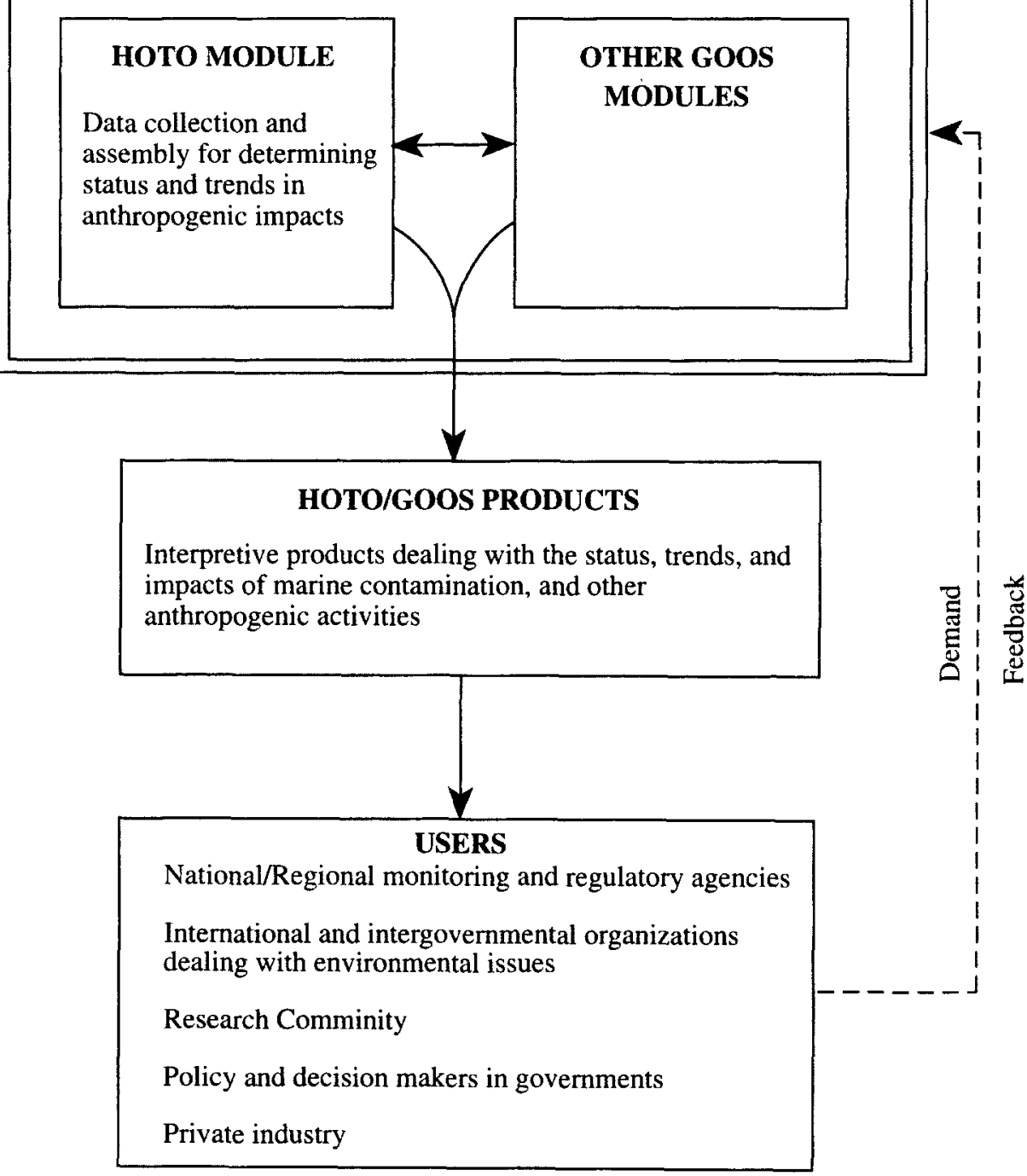

Fig. 4: Data delivery pathways.

sources are just some of the tools that will be part of assessing the health of the oceans.

\section{Activities to be Undertaken}

The areas of initial emphasis are recommended to be as follows:

1. Development of a set of reliable. relatively easily applicable biological stress indices reflecting the condition (i.e., health) of the marine environment;

2. Monitoring of the extent of habitat losses in coastal zones;

3. Monitoring of the effects of altered sediment loads on the coastal zones:

4. Monitoring concentrations and trends of contaminant loadings in coastal zones in relation to community responses; part require further research for full implementation in any observing system. These are

- Molecular and Cellular Biomarkers

- Cellular Pathology

- Physiological and Behavioral Responses

- Population and Community Monitoring

\section{GOOS Modular Interaction}

Each GOOS module can benefit from the receipt of data collected within other modules (see Fig. 4). There may be significant overlaps (i.e.. partial duplication) among the measurements required by individual modules and that, given the correct type and level of coordination, these can be minimized while at the same time offering increased economy and effectiveness. Similarly, there will be components of the interpretative products of individual modules that will be relevant to meeting the objectives and providing contributions to the interpretative products of other modules. Again. appropriate coordination within GOOS should ensure both economy and effectiveness in the aggregate operational activities of GOOS.

\section{Members of the Health of the Ocean Panel}

Dr. Neil Andersen, Univ. of Maryland, USA (Chairman)*

Dr. Samuel Anurigwo, Imo State EPA, Nigeria

Dr. J. Michael Bewers, Bedford Inst. of Ocean., Canada

Dr. Robert Bowen. Univ. of Massachusetts, USA

Prof. Michael Depledge. Univ. of Plymouth, U.K.

Prof. Edgardo Gomez, Univ. of the Philippines. Philippines

Prof. John Gray, Univ. of Oslo, Norway

Prof. Youssef Halim, Faculty of Science, Egypt

Dr. Michael Huber, John Cook Univ., Australia

Dr. Anthony Knap, Bermuda Biological Station, Bermuda Prof. Michael Moore, Plymouth Marine Laboratory, U.K.

* To whom inquiries should be made: Univ. of MD, Horn Point Environmental Laboratory-CEES, P.O. Box 775. Cambridge. MD 21613, USA: email (Internet) <andersen@ hpel. umd.edu>. 


\section{Dr. Md. Sirajul Islam, Int. Center for} Diarrhoel Desease Research, Bangladesh

Dr. Jonathan Patz, Johns Hopkins School of Hygiene and Public Health, USA

\section{References}

Fairweather, P.G., 1991: Statistical power and design requirements for environmental monitoring, Austral. J. Mar. Freshwater Res., 42, 555-567.

Fleming, N., 1995: Making the Case for GOOS. Sea Tech., Jan., 4 pp.

Gray, G.S. and K. Jensen, 1993: Feedback monitoring: a new way of protecting the environment. Trends Ecol. Evol., 8, 267-268.

IOC, 1976: A Comprehensive Plan for the Global Investigation of Pollution in the Marine Environment and Baseline Guidelines, Tech.
Series No. 14, Paris.

1984: A Framework for the Implementation of the Comprehensive Plan for the Global Investigation of Pollution in the Marine Environment, Tech. Series No. 25, Paris.

, 1991: Summary Report, Seventh Session of the IOC/UNEP/IMO Committee on the Global Investigation of Pollution in the Marine Environment, Paris, 21-25 January.

1995: Summary Report, First Session, Strategy Sub-Committee, Geneva, 27-30 March.

The Ocean Observing System Panel (OOSDP), 1995: Scientific Design for the Common Module of the Global Ocean Observing System and the Global Climate Observing System: An Ocean Observing System for Climate, Dept. Of Ocean., Texas A\&M Univ., 265 pp.

Peterman, R.M., 1990: Statistical power analysis can improve fisheries research and management. Can. J. Fish. Aquar. Sci., 47, 2-15.

Scholin, C., N. Wainwright and G. Hallegraeff,
1994: Feasibility of Developing a Rapid Diagnostic Test for Toxic Dinoflagellates in Ships' Ballast Water. Consultancy Rep., Australian Quarantine and Inspection Service, Dec.

$\longrightarrow$ K.R. Britschgi, J. Cangelosi, and F.P. Chavez, 1996: Identification of Pseudo-nitzshia australis (Bacillariophyceae) using rRNA-targeted probes in whole cell and sandwich hybridization formats. Phycologia 35, 190-197.

Underwood, A.J., 1991: Beyond BACI: Experimental designs for detecting human environmental impacts on temporal variations in natural populations. Australian. J. Mar. Freshwater Res., 42, 569-587.

1992: Beyond BACI: the detection of environmental impacts on populations in the real, but variable, world. J. Exp. Mar. Biol. Ecol., 161, 145-178.

UNEP Regional Seas Program, 1991: Compendium of Projects, UNEP Reg. Seas Rep. \& Studies, No. 19, Rev. 6, UNEP, Nairobi, 83 pp. 